(C) 2016 IEEE. Personal use of this material is permitted. Permission from IEEE must be obtained for all other uses, in any current or future media, including reprinting/republishing this material for advertising or promotional purposes, creating new collective works, for resale or redistribution to servers or lists, or reuse of any copyrighted component of this work in other works. 


\title{
Detection of Gait Initiation Failure in Parkinson's Disease Patients using EEG Signals
}

\author{
Quynh Tran Ly , A.M. Ardi Handojoseno $^{\mathrm{a}, \mathrm{c}}$, Student Member, IEEE, Moran Gilat ${ }^{\mathrm{b}}$, \\ Nghia Nguyen ${ }^{\text {, }}$ Senior Member, IEEE, Rifai Chai ${ }^{a}$, Member, IEEE, Yvonne Tran ${ }^{a}$,

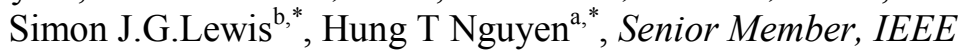

\begin{abstract}
Gait Initiation Failure (GIF) is one of the most disabling gait disturbances seen in advanced Parkinson's disease (PD). Gait Initiation is a complex motor task that requires motor and cognitive processing to enable the correct selection, timing and scaling of movement. Failure to initiate the first step often precipitates falls and leads to significant morbidity. However, the brain mechanisms underlying GIF remain unknown. This study utilized an ambulatory electroencephalography (EEG) technique to investigate the brain dynamic changes underlying GIF and aims to detect the occurrence of GIF in four PD patients. We sought to determine whether episodes of GIF might be associated with a characteristic brain signal that could be detected by surface EEG. This preliminary investigation analyzed the EEG signals through power spectra density (PSD) and centroid frequency (CF) to show that the GIF episodes were associated with significant increases in the high beta band $(21-38 \mathrm{~Hz})$ across the central, frontal, occipital and parietal EEG sites. By implementing PSD and CF as input features with two-layer Back Propagation neural networks as a classifier, the proposed system was able to detect GIF events with a classification performance of $84.27 \%$ sensitivity and $84.80 \%$ accuracy. This is the first study to show cortical dynamic changes associated with GIF in Parkinson's disease, providing valuable information to enhance the performance of future GIF detection that could be translated into clinical practice.
\end{abstract}

\section{INTRODUCTION}

Gait Initiation Failure (GIF) or "start hesitation" is a subtype of Freezing of Gait (FOG) seen in PD. Patients with GIF are frequently unable to initiate their first step effectively in order to start walking. GIF is a common form of FOG, with over 20 percent of all FOG episodes recorded as being a GIF [1]. Gait initiation is a complex motor task requiring both motor and cognitive processing to enable the correct selection, timing and scaling of movement [2]. Gait initiation in PD patients with FOG can be affected by emotion, attention and dopaminergic therapy, suggesting the existence

\footnotetext{
a Faculty of Engineering and Information Technology, University of Technology, Sydney, Broadway, NSW 2007, Australia. (Quynh.T.Ly@student.uts.edu.au,AluysiusMariaArdi.Handojoseno@studen t.uts.edu,Rifai.Chai@uts.edu.au,TuanNghia.Nguyen@uts.edu.au,yvonne.tra n@uts.edu.au, Hung.Nguyen@uts.edu.au)

b Parkinson's Disease Research Clinic, Brain and Mind Research Center, University of Sydney, Level 4, Building F, 94 Mallet Street, Camper down, NSW, 2050,

(simonl@med.usyd.edu.au,moran.gilat@sydney.edu.au)

${ }^{c}$ Faculty of Science and Engineering, Sanata Dharma University, Paingan, Sleman, Yogyakarta, 55281, Indonesia (ardi@usd.ac.id)

"Dual senior authorship
}

of a complex pathophysiological process that not only involves the locomotor networks, but also differing cortical areas and the basal ganglia system [2]. However, little objective evidence exists regarding the brain mechanisms underlying GIF and there are no clinically useful approaches for its treatment [3].

Our research group has recently demonstrated that EEG techniques can be used to identify and detect FOG episodes in PD due to their temporal ability to track the dynamic physiological changes throughout the brain during and even prior to the occurrence of any motor disturbances $[4,5]$. An early detection algorithm for FOG has already been developed by analyzing energy power, entropy, correlation and brain connectivity of EEG signals, providing valuable insights into the underlying brain mechanism of FOG, which are progressing the development of novel treatments $[4,5]$. However, these previous studies have focused on episodes of freezing in general without classifying subtypes of FOG, potentially limiting its clinical accuracy.

To the best of our knowledge, there is currently no implementation of EEG techniques to investigate and detect GIF in PD patients. In this study, we therefore used a noninvasive EEG system to study four PD patients who experienced multiple episodes of GIF during several standardized Timed Up and Go (TUG) assessments [1]. In this paper, the method of GIF detection is based on Back Propagation neural networks with the input features being Power Spectral Density (PSD) and Centroid Frequency (CF) $[4,5]$.

Based on the notion that gait initiation requires both motor and non-motor features (e.g. cognitive and limbic processes), we aimed to investigate neural changes associated with GIF. We hypothesized that there would be a detectable change in high beta CF and PSD across the central and frontal regions when comparing GIF with effective starts as high beta frequencies from the frontal lobe have been proposed to act as "a stopping signal" that might prevents the central motor area's from initiating the first step [6]. We were also keen to explore whether the classification results could be detected using only two channels; which would contribute to the feasibility of making real-time devices for clinical application.

\section{METHODS}

\section{A. Subjects and Task}

EEG data were obtained from four Parkinson's disease patients ( 3 males, 1 female) with clinically confirmed FOG. They were recruited from the Parkinson's disease Research Clinic at the Brain and Mind Centre, The University of 
Sydney. This study was approved by The Human Research and Ethics Committee from the University of Sydney. They were assessed in their practically-defined 'off' state following overnight withdrawal of dopaminergic therapy for more than 12 hours. All subjects demonstrated multiple episodes of GIF during a structured series of video-recorded TUG tasks. The distribution of GIF among the patients was not equal: 3 patients had less than 15 events and 1 patient experienced more than 15 events. As such, we randomly selected 15 GIF events from this subject to be used in further analyses. The EEG data were acquired using a $32 \mathrm{Ag} / \mathrm{AgCl}$ scalp electrodes of a Biosemi ActiveTwo system. Only data from 9 electrodes positioned in the location of interest were processed: F3, Fz, F4 (motor planning and working memory), $\mathrm{Cz}$ (motor execution), P3, Pz, P4 (sensory integration), $\mathrm{O} 1$ and $\mathrm{O} 2$ (visual area). References were taken by averaging from electrodes placed on each ear lobe. The recording was segmented into 1-second durations and digitized at $512 \mathrm{~Hz}$.

The EEG data of GIF events was taken according to the time of onset and offset as scored on the video when the patients tried to take a first step but failed to do so. In addition, the EEG data from Good Starts (GS) was taken from 2-second periods after the patients were able to take an effective first step in a normal start during the TUG tasks.

\section{B. Feature Extraction}

In this study, 61 GIF EEG and 61 GS EEG samples data were collected from four patients and analyzed using EEGLab toolbox. The EEG signals were filtered using a nonlinear IIR band-pass filter with a cut-off frequency lower than $1 \mathrm{~Hz}$ and higher than $50 \mathrm{~Hz}$ to remove artifacts. The amplitude spectrum of each data sample in the time domain was transformed into the frequency domain using Fast Fourier Transform; which resulted in the power spectrum $P$ $\left(f_{i}\right)$. It has been proposed that beta band activity can be further subdivided into low and high frequency, with high beta frequencies being affected differently in response to FOG [7]. Therefore, in this study, Welch's method with a 256 points FFT with $55 \%$ overlapping was used to analyze four frequency sub-bands, namely theta $(4-8 \mathrm{~Hz})$, alpha $(8-13 \mathrm{~Hz})$, low beta (13-21 Hz) and high beta (21-38Hz) (Fig. 1).

The PSD and CF of each frequency band were estimated and chosen as the main parameters for further analyses. The non-parametric Wilcoxon Sum Rank Test with an alpha of 0.05 was used to investigate the PSD and CF differences between GS and GIF episodes. These features were then used as inputs of classifier for the detection of GIF.

Fig. 1 shows the amplitude spectra of representative raw EEG data of one patient that were tracked in the time domain. Overall, decomposition of the EEG data into theta, alpha and low-high beta bands demonstrated that GIF was associated with high amplitude within a range from 4 to $8 \mathrm{~Hz}$ (theta) and 21 to $38 \mathrm{~Hz}$ (high beta). In addition, GS was typically characterized by regularly decreased amplitude in these two sub-bands within different brain regions.

\section{Classification}

In this paper, we utilized a two-layer feed-forward neural networks with 2 to 12 hidden nodes to classify the pattern into two categories: GIF and GS. The input of this classifier

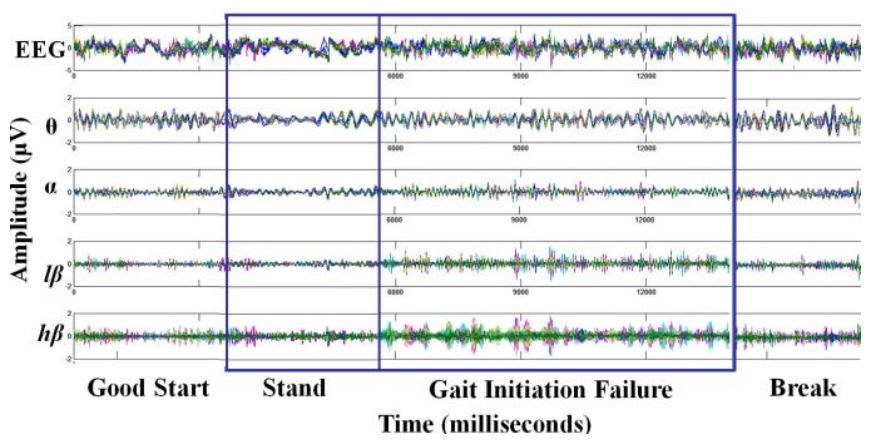

Figure 1. The comparison of amplitude and frequency of raw EEG data between GS, Stand, GIF and Break (Breaking GIF) in one PD patient.

included PSD and CF features extracted from the EEG signals. The desired output was set at 1 in cases of GIF and 0 in cases of GS (Fig. 2).

The output of the neural networks was computed as follows:

$$
z(x, w)=f_{1}\left(b_{k}+\sum_{j=1}^{m} \bar{w}_{k j} f_{2}\left(b_{j}+\sum_{i=1}^{n} w_{j i} x_{n}\right)\right)
$$

where $f_{1}, f_{2}$ is the activation function, $x$ presents the input vector, $W$ is the weight matrix vector; $w_{j i}$ is the weight of the link between the $i$-th hidden node and the $j$-th input; $\bar{w}_{k j}$ is the weight of the link between $i$-th hidden node and the output, $b_{\mathrm{k}}$ and $b_{\mathrm{j}}$ are the biases; $m$ is the number of outputs; $n$ is the number of inputs and the tan sig function was assigned as the activation function of the hidden layer.

For the classification purpose, $122 \mathrm{EEG}$ data (including $61 \mathrm{GS}$ and $61 \mathrm{GIF}$ ) from 4 PD patients were fed into our classifier. Levenberg-Marquardt algorithm was chosen as training method for its speed and stability. To avoid overtraining, our classification system utilized early stopping strategy that helped preventing over-fitting and improved generalization. The data set was separated randomly into training set, validation set and test set with the ratio of $34 \%$, $33 \%$ and $33 \%$, respectively. The prediction of sensitivity, specificity and accuracy were measured based on 50 times trials.

\section{RESULTS}

\section{A. EEG Power Spectral Density}

Statistical results of extracted PSD of EEG signal at each

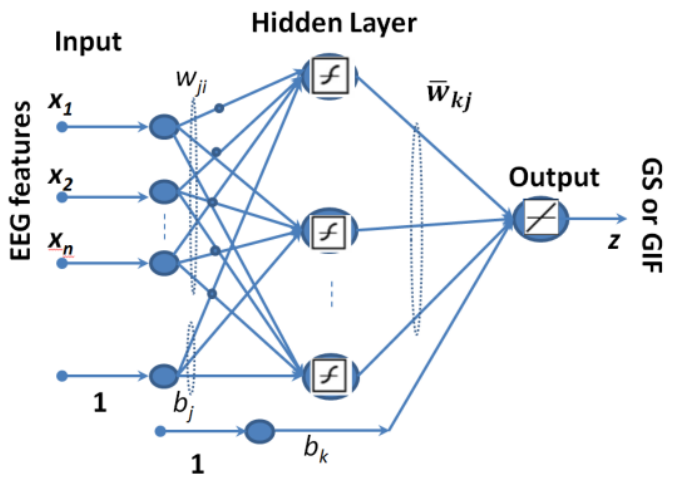

Figure 2. Neural Networks Structure 
single channel are presented in Table I, with the most significant features $(\boldsymbol{p}<\mathbf{0 . 0 0 1})$ being reported in italic bold.

Direct comparison of EEG frequency band power during two conditions showed that the strongest significant differences were found in the beta band, especially high beta in the occipital areas $\mathrm{O} 1$ and $\mathrm{O} 2(p<0.0001)$. In addition, the results revealed that the parietal areas $\mathrm{P} 3$ and $\mathrm{P} 4$ were significantly affected by GIF in the high beta frequency band $(p<0.001)$. We also observed a significant increase in both low and high beta power during GIF in the central $\mathrm{Cz}$ and frontal $\mathrm{Fz}$ areas.

In Table II, the comparison of CF during the two conditions (e.g. GS vs GIF) showed that CF high beta stood out as the only affected sub-band during GIF regardless the position of the EEG channel. More specifically, the CF high beta increased significantly in almost 8 channels during GIF episodes. This pattern indicated that GIF may be related to a change in the oscillatory high beta frequency within the subthalamic nucleus and may also support the suggestion that GIF is related to excessive paroxysmal cortical beta synchronization [7]

Overall, in this finding, we proposed that the changes of oscillatory rhythms during GIF reflected an increase in conflict processing underlying cognitive control (theta) and "stopping" network operating (high beta) within the motor planning and execution in the centers of the brain $[5,7,8]$.

TABLE I. POWER SPECTRAL DENSITY BETWEEN GIF AND GS

\begin{tabular}{|c|c|c|c|c|}
\hline Lead & band & GS & GIF & $p$-value \\
\hline F3 & $\theta$ & $0.130 \pm 0.15$ & $0.162 \pm 0.13$ & 0.0313 \\
\hline \multirow{4}{*}{$\mathrm{Fz}$} & $\theta$ & $0.129 \pm 0.11$ & $0.191 \pm 0.15$ & $\leq 0.001$ \\
\hline & $\alpha$ & $0.057 \pm 0.05$ & $0.063 \pm 0.04$ & 0.0425 \\
\hline & $1 \beta$ & $0.030 \pm 0.03$ & $0.036 \pm 0.02$ & 0.0161 \\
\hline & $\mathrm{h} \beta$ & $0.025 \pm 0.03$ & $0.033 \pm 0.03$ & 0.0471 \\
\hline \multirow{3}{*}{$\mathrm{Cz}$} & $\theta$ & $0.128 \pm 0.13$ & $0.172 \pm 0.14$ & 0.0132 \\
\hline & $1 \beta$ & $0.032 \pm 0.02$ & $0.044 \pm 0.03$ & 0.0245 \\
\hline & $h \beta$ & $0.032 \pm 0.03$ & $0.049 \pm 0.04$ & $\leq 0.001$ \\
\hline \multirow{3}{*}{ P3 } & $\theta$ & $0.130 \pm 0.15$ & $0.162 \pm 0.13$ & 0.0328 \\
\hline & $l \beta$ & $0.034 \pm 0.03$ & $0.046 \pm 0.03$ & $\leq 0.001$ \\
\hline & $h \beta$ & $0.043 \pm 0.05$ & $0.069 \pm 0.07$ & $\leq 0.001$ \\
\hline \multirow{2}{*}{ P4 } & $1 \beta$ & $0.036 \pm 0.03$ & $0.048 \pm 0.03$ & 0.0101 \\
\hline & $h \beta$ & $0.044 \pm 0.04$ & $0.073 \pm 0.06$ & $\leq 0.001$ \\
\hline \multirow{3}{*}{$\mathrm{Pz}$} & $\theta$ & $0.132 \pm 0.14$ & $0.171 \pm 0.14$ & 0.0377 \\
\hline & $1 \beta$ & $0.034 \pm 0.03$ & $0.044 \pm 0.03$ & 0.0090 \\
\hline & $\mathrm{h} \beta$ & $0.035 \pm 0.03$ & $0.055 \pm 0.06$ & 0.0100 \\
\hline \multirow{3}{*}{$\mathrm{O} 1$} & $\alpha$ & $0.056 \pm 0.05$ & $0.072 \pm 0.05$ & 0.0139 \\
\hline & $l \beta$ & $0.045 \pm 0.04$ & $0.069 \pm 0.05$ & $\leq 0.001$ \\
\hline & $h \beta$ & $0.077 \pm 0.08$ & $0.134 \pm 0.10$ & $\leq 0.0001$ \\
\hline \multirow{4}{*}{$\mathrm{O} 2$} & $\theta$ & $0.094 \pm 0.10$ & $0.110 \pm 0.08$ & 0.0280 \\
\hline & $\alpha$ & $0.059 \pm 0.05$ & $0.089 \pm 0.06$ & $\leq 0.001$ \\
\hline & $l \beta$ & $0.046 \pm 0.04$ & $0.083 \pm 0.06$ & $\leq 0.0001$ \\
\hline & $h \beta$ & $0.081 \pm 0.08$ & $0.139 \pm 0.12$ & $\leq 0.0001$ \\
\hline
\end{tabular}

TABLE II. CENTROID FREQUENCY BETWEEN GIF AND GS

\begin{tabular}{ccccc}
\hline Lead & band & GS & GIF & $\boldsymbol{p}$-value \\
\hline $\mathrm{F} 3$ & $\mathrm{~h} \beta$ & $25.826 \pm 1.94$ & $26.461 \pm 1.92$ & 0.0307 \\
$\mathrm{Fz}$ & $\mathrm{h} \beta$ & $25.682 \pm 1.81$ & $26.247 \pm 1.89$ & 0.0428 \\
\hline $\mathrm{Cz}$ & $\mathrm{h} \beta$ & $25.858 \pm 2.05$ & $26.585 \pm 1.82$ & 0.0166 \\
\hline $\mathrm{P} 3$ & $\mathrm{~h} \beta$ & $26.287 \pm 2.15$ & $27.168 \pm 2.06$ & 0.0107 \\
$\mathrm{P} 4$ & $\mathrm{~h} \beta$ & $26.219 \pm 2.14$ & $27.041 \pm 1.97$ & 0.0158 \\
$\mathrm{Pz}$ & $\mathrm{h} \beta$ & $26.383 \pm 2.29$ & $27.182 \pm 1.94$ & 0.0211 \\
\hline $\mathrm{O} 1$ & $\mathrm{~h} \beta$ & $26.596 \pm 2.23$ & $27.658 \pm 2.03$ & 0.0300 \\
$\mathrm{O} 2$ & $\mathrm{~h} \beta$ & $26.826 \pm 2.41$ & $27.591 \pm 1.94$ & 0.0258 \\
\hline
\end{tabular}

\section{B. Classification}

The classification result displays consistent values are shown in Fig. 3. The classification percentages shows consistent values in all fifty running times confirmed the ability of the optimization process to provide the optimal solution in each of the regions. The number of hidden nodes was varied from 2 to 12 per training session of the neural networks for each separate location in order to find the best number of hidden nodes that provides the highest sensitivity and best accuracy.

The mean, maximum and minimum values of PSD and CF of the 4 frequency bands in each electrode's location were taken as inputs of the classifier to evaluate their strength in detecting GIF. The sensitivity, specificity and accuracy results of our classification system are shown in Table III-IV. The reported results were the best performance (highest sensitivity and best accuracy) between 2 and 12 hidden nodes and the mean of 50 times trials. We also studied the performance of the detection of GIF using the combination of these different channels. The neural networks were developed with inputs corresponding to significant data $(\mathrm{p}<0.05)$ from one, two, four and nine EEG channels.

The classifier system using input from single locations was represented in Table III. The result showed that occipital regions provided the best location data for detecting GIF events with classifier using data from channel O2 obtained $70.90 \%$ sensitivity and $74.44 \%$ accuracy. Central locations provided similar strength as an indicator of GIF with $\mathrm{Cz}$ detected GIF with $68.29 \%$ sensitivity and $74.44 \%$ accuracy. The best performance of classifier using single channel from frontal and parietal locations was when

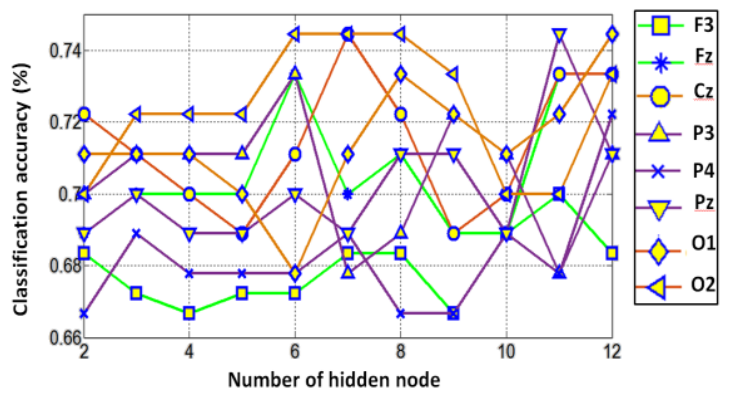

Figure 3. Mean of accuracy versus number of hidden nodes. 
TABLE III. CLASSIFICATION RESULTS OF PROPOSED FEATURES USING BACK PROPAGATION NEURAL NETWORKS

\begin{tabular}{cccccccc}
\hline \multirow{2}{*}{ Lead } & \multirow{2}{*}{ H } & \multicolumn{3}{c}{ Training set } & \multicolumn{3}{c}{ Testing set } \\
\cline { 3 - 8 } & & Sens & Spec & Acc & Sens & Spec & Acc \\
\hline F3 & 10 & 70.37 & 76.42 & 73.26 & 67.78 & 69.72 & 68.89 \\
Fz & 12 & 78.84 & 84.32 & 81.63 & 72.22 & 72.89 & 73.33 \\
\hline Cz & 7 & 74.99 & 85.61 & 80.00 & 68.29 & 79.55 & 74.44 \\
\hline P3 & 2 & 71.72 & 82.16 & 76.51 & 69.84 & 69.70 & 70.00 \\
P4 & 3 & 72.21 & 71.01 & 71.63 & 69.29 & 68.55 & 68.89 \\
Pz & 9 & 71.70 & 84.92 & 77.91 & 68.29 & 72.88 & 71.11 \\
\hline O1 & 9 & 75.36 & 85.27 & 80.23 & 70.95 & 72.16 & 72.22 \\
O2 & 6 & 76.63 & 93.69 & 84.65 & 70.90 & 77.06 & 74.44 \\
\hline
\end{tabular}

$\mathrm{H}=$ number of hidden nodes; Sens $=$ sensitivity; Spec $=$ specificity; Acc $=$ accuracy;

using $\mathrm{Fz}$ and $\mathrm{Pz}$ with $72.22 \%$ and $68.29 \%$ sensitivity, respectively. These two locations achieved $73.33 \%(\mathrm{Fz})$ and $71.11 \%(\mathrm{Pz})$ accuracy in detecting GIF.

$\mathrm{CzO} 2$ appeared at the best combination of two channels to detect GIF with sensitivity 76.88\% and accuracy $78.89 \%$, followed by $\mathrm{FzO} 2$ with sensitivity of $71.67 \%$ and accuracy of $78.89 \%$. The combination of four best channels FzCzPzO2 obtained with $78.50 \%$ sensitivity and $82.22 \%$ accuracy when detecting GIF. The best performance of the classification system was $84.27 \%$ sensitivity and $84.80 \%$ accuracy in using a combination of nine channels (Table IV).

\section{DISCUSSION}

Abnormal beta band frequencies were found underlying GIF. This finding is consistent with previous research showing that beta frequencies are likely to operate the neural communications in a stopping network in which the beta power increases when the prohibiting of movement is needed [8]. In addition, a relationship between freezing and high-beta oscillations in the subthalamic nucleus has been suggested with maximal coherence in high beta activity being located across the supplementary motor cortex (SMA), cingulate and leg area of SMA [7]. Finally, our research group recently found that beta frequencies are the most significant features of EEG signals underlying the transition from walking to FOG $[4,5]$.

Overall, these results are closely aligned with our current finding, suggesting that the high beta oscillations observed over central and frontal regions $(\mathrm{Cz}-\mathrm{Fz})$ of the cortex could represent inhibitory signals from the basal ganglia and its output nuclei, which are likely to be associated with the inability to start walking during GIF in PD patients [7, 8].

TABLE IV. CLASSIFICATION RESULTS OF COMBINATION OF CHANNELS

\begin{tabular}{cccccccc}
\hline \multirow{2}{*}{ Lead } & \multirow{2}{*}{ H } & \multicolumn{3}{c}{ Training set } & \multicolumn{3}{c}{ Testing set } \\
\cline { 3 - 8 } & & Sens & Spec & Acc & Sens & Spec & Acc \\
\hline $\mathrm{FzCz}$ & 2 & 71.14 & 91.20 & 80.93 & 67.62 & 80.81 & 75.56 \\
$\mathrm{FzPz}$ & 5 & 76.81 & 85.88 & 80.93 & 70.56 & 74.56 & 73.33 \\
$\mathrm{FzO} 2$ & 11 & 76.87 & 94.64 & 85.35 & 71.67 & 84.46 & 78.89 \\
\hline $\mathrm{CzPz}$ & 11 & 74.87 & 88.51 & 81.86 & 71.16 & 74.98 & 73.33 \\
$\mathrm{CzO} 2$ & 8 & 80.26 & 96.57 & 88.37 & 76.88 & 81.92 & 78.89 \\
\hline $\mathrm{PzO} 2$ & 7 & 70.96 & 96.22 & 83.49 & 68.95 & 87.07 & 78.89 \\
\hline FzPzCzO2 & 12 & 81.78 & 97.14 & 89.07 & 78.50 & 85.38 & 82.22 \\
FCPO & 10 & 94.85 & 87.75 & 90.34 & 84.27 & 85.02 & 84.80 \\
\hline FCPO: F3Fz-Cz-P3PzP4-O1O2 & & & &
\end{tabular}

The classification results suggest that central-occipital $(\mathrm{CzO} 2)$ cortex regions are the two optimal locations for detecting GIF in PD patients. This indicates that the PD patients are 'over-relying' on visual information during a GIF. Especially during a period of GIF, due to the inadequate input from motor regions $(\mathrm{Cz})$, the response of the muscle and joints in PD patients are different than what they are expected. Therefore, these freezers might need to use more of their visual system $(\mathrm{O} 2)$ to gain the information about what is happening in order to break GIF by getting $\mathrm{Cz}$ (primary motor regions) to activate again [9].

\section{CONCLUSION}

This study revealed that high beta frequency band stood out as the most effected sub-band underlying GIF behaviour in PD patients. The classification showed that centraloccipital might be the most two impaired regions during the GIF episode. Results also showed the episode of GIF can be detected using only two channels with average accuracy above $75 \%$. Future studies using larger sample sizes that compare other multiple electrode sites are now required to confirm our proposed methodology. In addition, future studies are encouraged to continue to pursue the development of a real-time device that can efficiently predict GIF events to alert PD patients when GIF is detected.

\section{REFERENCES}

[1] J. M. Shine, S. T. Moore, S. J. Bolitho, T. R. Morris, V. Dilda, S. L. Naismith, et al., "Assessing the utility of Freezing of Gait Questionnaires in Parkinson's Disease," Parkinsonism \& Related Disorders, vol. 18, pp. 25-29, 2012.

[2] A. Delval, C. Moreau, S. Bleuse, D. Guehl, E. Bestaven, E. Guillaud, et al., "Gait and attentional performance in freezers under methylphenidate," Gait \& Posture, vol. 41, pp. 384-388, 2015.

[3] K. Niazmand, K. Tonn, Y. Zhao, U. M. Fietzek, F. Schroeteler, K. Ziegler, et al., "Freezing of Gait detection in Parkinson's disease using accelerometer based smart clothes," in Biomedical Circuits and Systems Conference (BioCAS), 2011 IEEE, pp. 201-204, 2011.

[4] A. M. Ardi Handojoseno, J. M. Shine, T. N. Nguyen, Y. Tran, S. J. G. Lewis, and H. T. Nguyen, "Analysis and Prediction of the Freezing of Gait Using EEG Brain Dynamics," Neural Systems and Rehabilitation Engineering, IEEE Transactions on, vol. 23, pp. 887-896, 2015.

[5] J. M. Shine, A. M. A. Handojoseno, T. N. Nguyen, Y. Tran, S. L. Naismith, H. Nguyen, et al., "Abnormal patterns of theta frequency oscillations during the temporal evolution of freezing of gait in Parkinson's disease," Clinical Neurophysiology, vol. 125, pp. 569576, 2014.

[6] A. R. Aron, T. W. Robbins, and R. A. Poldrack, "Inhibition and the right inferior frontal cortex: one decade on," Trends in Cognitive Sciences, vol. 18, pp. 177-185, 2014.

[7] J. B. Toledo, J. López-Azcárate, D. Garcia-Garcia, J. Guridi, M. Valencia, J. Artieda, et al., "High beta activity in the subthalamic nucleus and freezing of gait in Parkinson's disease," Neurobiology of Disease, vol. 64, pp. 60-65, 2014

[8] A. A. Kühn, F. Kempf, C. Brücke, L. Gaynor Doyle, I. MartinezTorres, A. Pogosyan, et al., "High-Frequency Stimulation of the Subthalamic Nucleus Suppresses Oscillatory $\beta$ Activity in Patients with Parkinson's Disease in Parallel with Improvement in Motor Performance," The Journal of Neuroscience, vol. 28, pp. 6165-6173, 2008

[9] K. A. Ehgoetz Martens, F. Pieruccini-Faria, and Q. J. Almeida, "Could Sensory Mechanisms Be a Core Factor That Underlies Freezing of Gait in Parkinson's Disease?," PLoS ONE, vol. 8, p. e62602, 2013 